\title{
Acondicionamiento previo deportivo para el Taekwondo Olímpico: una propuesta a partir de los ejercicios
}

Sports pre-conditioning for Olympic Taekwondo: a proposal as of the exercises

\author{
Luciana Angiolillo \\ Departamento de Educación Física, Facultad de \\ Humanidades y Ciencias de la Educación. Universidad \\ Nacional de La Plata, Argentina \\ angiolillo.luciana@gmail.com
}

\author{
Adrián Casas \\ Departamento de Educación Física, Facultad de \\ Humanidades y Ciencias de la Educación. Universidad \\ Nacional de La Plata, Argentina \\ profcasas@yahoo.com.ar
}

\section{Resumen:}

El Taekwondo Olímpico o Taekwondo Mundial (WT) es considerado el arte marcial más practicado del planeta, lidera el deporte de combate más inclusivo y accesible con una presencia activa de 210 asociaciones nacionales del mundo. Estos datos nos permiten dimensionar su importancia dentro del deporte a nivel mundial. La teoría y práctica del entrenamiento deportivo, destaca la importancia de diversas herramientas para la mejora del rendimiento del deportista. Las actividades realizadas al inicio de cada sesión de entrenamiento o competición, han demostrado influir positivamente en el rendimiento inmediato. El conjunto de estas actividades, se denomina acondicionamiento previo deportivo. En este artículo, abordaremos -desde la estructura del acondicionamiento previo deportivo-, una clasificación de los ejercicios según criterios fisiológicos y mecánicos, con la finalidad de analizar, identificar y proponer los ejercicios más idóneos para el acondicionamiento previo deportivo del Taekwondo Olímpico. Palabras clave: Calentamiento, Acondicionamiento previo deportivo, Ejercicio, Taekwondo, Deportes de combate.

\section{ABstract:}

Olympic Taekwondo or World Taekwondo (WT) is considered the most practiced martial art on the planet, leading the most inclusive and accessible combat sport with an active presence of 210 national associations in the world. These data allow us to measure its importance within the sport sphere at a global level. The theory and practice of sports training highlight the importance of various tools for improving the athlete's performance. The activities carried out at the beginning of each training session or competition have shown positive influence in immediate performance. All of these activities are called sports previous conditioning. In this article, we will depart from the structure of previous conditioning and deal with a classification of exercises according to physiological and mechanical criteria, with the purpose of analyzing, identifying and proposing the most suitable exercises for the Olympic Taekwondo's sports previous -conditioning.

KEYWORDS: Warm up, Sports previous conditioning, Exercise, Taekwondo, Combat sports.

\section{INTRODUCCIÓN.}

El Taekwondo Olímpico combina los valores de una antigua herencia asiática con los de un deporte de élite global. Más de 60.000 deportistas de todo el planeta practican la modalidad de combate, liderando así, el listado de las artes marciales más practicadas del mundo. Ello nos permite dimensionar su importancia dentro del deporte a nivel mundial. 
La teoría y práctica del entrenamiento deportivo, destaca la importancia de diversas herramientas para la mejora del rendimiento del deportista. Las actividades realizadas al inicio de cada sesión de entrenamiento o competición, han demostrado influir positivamente en el rendimiento inmediato. El conjunto de estas actividades, fue denominado - tradicionalmente - "entrada en calor" o "calentamiento", ambos términos alcanzan decenas de miles de resultados en una búsqueda en la web.

El objetivo de este trabajo es proponer los ejercicios más idóneos para el acondicionamiento previo deportivo (APD) del Taekwondo Olímpico. Comenzamos el artículo presentando una denominación más precisa, APD, en reemplazo de las tradicionales. Luego, analizamos su estructura y desde criterios fisiológicos y mecánicos, estudiamos diferentes ejercicios con la finalidad de identificarlos y proponerlos para el acondicionamiento previo deportivo del Taekwondo Olímpico.

\section{ACONDICIONAMIENTO PREVIO DEPORTIVO.}

El calentamiento previo, según el Colegio Americano de Medicina del Deporte (ACSM, 2012), consiste en realizar ejercicios de baja intensidad que preparan el cuerpo para una actividad física más intensa. Es una práctica que precede a casi todos los eventos deportivos y se considera esencial para un rendimiento óptimo. El concepto, habitualmente utilizado, "entrada en calor" o "calentamiento" sugiere el uso de actividades cuyo objetivo refiere al incremento de la temperatura como factor central. Sin embargo, el rendimiento deportivo óptimo depende de una amplia gama de componentes que no están relacionados sólo con el incremento de la temperatura. De modo que, proponemos el concepto “acondicionamiento previo deportivo" (APD) en reemplazo de los anteriores ya que considera otras dimensiones (físicas, psicológicas, volitivas, etc.) del rendimiento deportivo y resulta más preciso y específico. Casas propone que:

"El acondicionamiento previo es el conjunto de actividades dirigidas a preparar al individuo y/o grupo para desarrollar una prestación o rendimiento deportivo eficaz. La preparación incluye el plano psicológico y fisiológico. Se destacan, además, aspectos técnicos específicos y volitivos como también los ajustes funcionales relacionados con el estrés de la competencia” (2008, p. 31).

\section{ACONDICIONAMIENTO PREVIO DEPORTIVO, SU UBICACIÓN DENTRO DE LA ESTRUCTURA DEL ENTRENAMIENTO DEPORTIVO.}

La integridad del proceso de entrenamiento se asegura sobre la base de una estructura determinada, la cual posee un orden relativamente estable de unión entre sus componentes, la correlación lógica de uno con el otro y una sucesión general (Matveiev, 1985). En la figura 1, podemos observar la estructura del proceso de entrenamiento y sus componentes. 
FIGURA 1.

Estructura del entrenamiento deportivo.

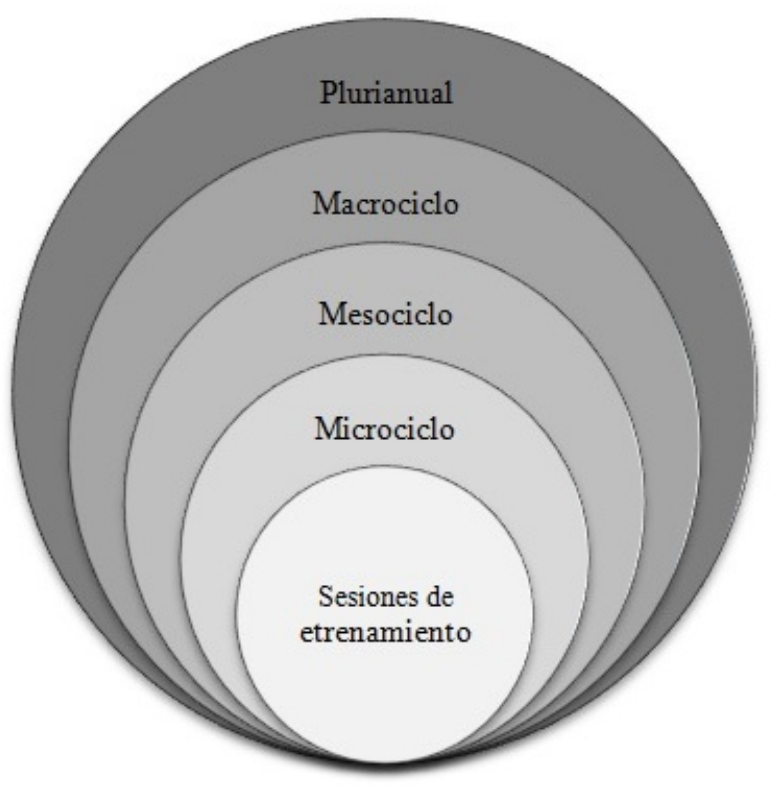

Fuente: elaboración propia.

La teoría del entrenamiento deportivo propone estas estructuras con el fin de adaptar al organismo a los esfuerzos; generar cambios en la condición física del deportista y mejoras de sus resultados deportivos. La sesión de entrenamiento es considerada la estructura más pequeña del proceso de organización y, a la vez, un integrante fundamental. Se compone de una fase inicial, otra central y la fase final. El APD se desarrolla en la fase inicial de toda sesión de entrenamiento, allí se prepara cognitiva, física y anímicamente al deportista, haciéndolo consciente del objetivo, de la exigencia y de la colaboración que se espera de él. En esta fase inicial, se concreta la preparación del organismo para la fase siguiente (entrenamiento o competición) de la sesión. El APD contribuye al desarrollo general del deportista, prepara el cuerpo para la alta intensidad y/o ejercicio de gran volumen, y reduce el riesgo potencial de lesiones. Generalmente, incluye algunos ejercicios aeróbicos y otros que aumentan el rango de movimiento de las articulaciones (ROM) como estiramientos, calistenia dinámica, y ejercicios específicos para cada deporte (ACSM, 2012).

En algunos casos, el APD es considerado un asunto secundario o marginal, lo cual no sólo constituye un grave error metodológico sino de contenido del entrenamiento, ya que éste produce efectos acumulativos y adaptativos en la mejora de la condición física del deportista. Por lo tanto, es considerado un elemento que contribuye a la carga del entrenamiento, potenciando y configurándola.

El APD está conformado por tres fases. La primera se denomina fase general, es la encargada de incrementar el potencial funcional del cuerpo (Siff \& Verkhoshasky, 2004). Se centra en aumentar los parámetros fisiológicos claves, sobre todo la distribución del flujo sanguíneo y la temperatura del cuerpo. Es importante que los ejercicios elegidos alcancen un tiempo adecuado de actividad. Es por ello que, una duración de 5 a 10 minutos (como mínimo) es apropiada. A continuación, la fase específica promueve la reducción de la viscosidad (muscular y articular), el incremento de la flexibilidad y el aumento de la temperatura muscular específica. Para esta fase, una duración de 10 a 15 minutos (mínimo) resulta apropiada. Por último, la fase aplicativa que impulsa un ajuste perceptivo motriz de los movimientos deportivos para la competencia y el entrenamiento. Mejora aspectos de la actividad deportiva que son esenciales para el rendimiento, como la coordinación específica, velocidad, agilidad y contracción pliométrica. Para esta fase, una duración de 10 a 15 minutos es adecuada. 


\section{Criterios de Clasificación de LOS EJERCiCios.}

Los ejercicios físicos son los elementos, la estructura y los medios principales del entrenamiento (Berger \& Hauptmann, 1996). Su importancia está dada porque su efecto tiene una relación directa con los factores de objetivación de la carga: volumen, intensidad, calidad de la ejecución motora, duración del entrenamiento (Harre, 1987; Berger \& Hauptman, 1996). Por lo tanto, es muy importante caracterizar al ejercicio de tal forma que se preste a la solicitación funcional del organismo para la cual el entrenador plantea dicha carga de entrenamiento.

Proponemos dos criterios para clasificar a los ejercicios, uno de ellos es fisiológico y el otro mecánico. El primero, denomina a los ejercicios: cardiovasculares (o de resistencia) y neuromusculares (o de fuerza). Es decir, los clasifica según los ejercicios provoquen mayor activación y sobrecarga en los sistemas cardiovascular o neuromuscular, respectivamente (Coffey \& Hawley, 2007). El segundo criterio, considera la correspondencia o relación cinética y cinemática que existe entre el ejercicio y la acción deportiva especifica. De este modo, la teoría del entrenamiento deportivo clasifica los ejercicios en generales o auxiliares, específicos o especiales y deportivos (Kuznetsov, 1975; Bompa, 1993; Derenne, Ho \& Murphy, 2001).

- Ejercicios generales o auxiliares: son aquéllos que mejoran el rendimiento físico en general y cuya mecánica de ejecución difiere del gesto deportivo.

- Ejercicios específicos o especiales: comprenden patrones motrices del deporte y se realizan en situaciones diferentes a las de competición.

- Ejercicios deportivos: son los que incluyen los mismos gestos deportivos, no sólo a nivel mecánico sino también las condiciones de espacio, situación y reglamento de competición.

El APD utiliza tanto ejercicios cardiovasculares como neuromusculares, generales, específicos y deportivos. Cada uno de ellos está relacionado con la fase del APD.

\section{Propuesta de ejercicios para el APD del Taekwondo Olímpico.}

Fisiológicamente, el Taekwondo Olímpico se caracteriza por la realización de esfuerzos rápidos y explosivos con acciones que incluyen numerosos golpes, bloqueos, saltos y desplazamientos. Las técnicas de patadas, bloqueos, ataques y otras acciones realizadas con explosividad durante la práctica del Taekwondo Olímpico son fuertemente dependientes del ciclo estiramiento-acortamiento y exigen un amplio rango de movilidad articular en miembros inferiores (Bridge, Ferreira da Silva Santos, Chaabéne, Pieter \& Franchini, 2014). Estas acciones, requieren de ejercicios dinámicos que producen un ajuste fisiológico en las distintas capacidades físicas (incremento de la fuerza y potencia, velocidad y agilidad, resistencia, flexibilidad y coordinación). El conjunto de estas actividades, constituye el APD y se desarrolla de manera gradual en intensidad, duración, dificultad y especificidad.

La estrategia de ejercicios que utilizamos para un APD efectivo y eficiente consiste en ejercicios generales, específicos y deportivos, de tipo cardiovascular y neuromuscular (Tabla 1). Además, en la Tabla 2, incluimos una breve descripción de cada uno de ellos.

La fase general incluirá ejercicios cardiovasculares y neuromusculares auxiliares, de baja a moderada intensidad. Incorpora ejercicios de estiramiento y ejercicios para activar el centro del cuerpo (core). Por ejemplo, carrera, skipping, burpees, saltos a la soga, carioca, spiderman, escalador, oruga, diferentes patrones de zancadas, sentadillas, marcha con miniband, planchas, puente de glúteos, etc.

La fase específica utiliza ejercicios de moderada a alta intensidad, que involucra al sistema nervioso central a través de movimientos relacionados con la especificidad deportiva. Incluye ejercicios dinámicos que implican 
patrones motrices Poliarticulares. Por ejemplo, desplazamientos, medios pateos, movimientos balísticos, salto en el lugar con caída unipodal, estocada frontal y medio pateo, distintos juegos de combate, etc.

La fase aplicativa incluye ejercicios deportivos, donde la intensidad alcanza niveles máximos similares a la competencia. Por ejemplo, combate reducido, pateos a diferentes focos (escudo, palmeta, etc.), diferentes secuencias de acciones técnicas y tácticas, etc.

TABLA 1

Ejercicios según criterio fisiológico y mecánico.

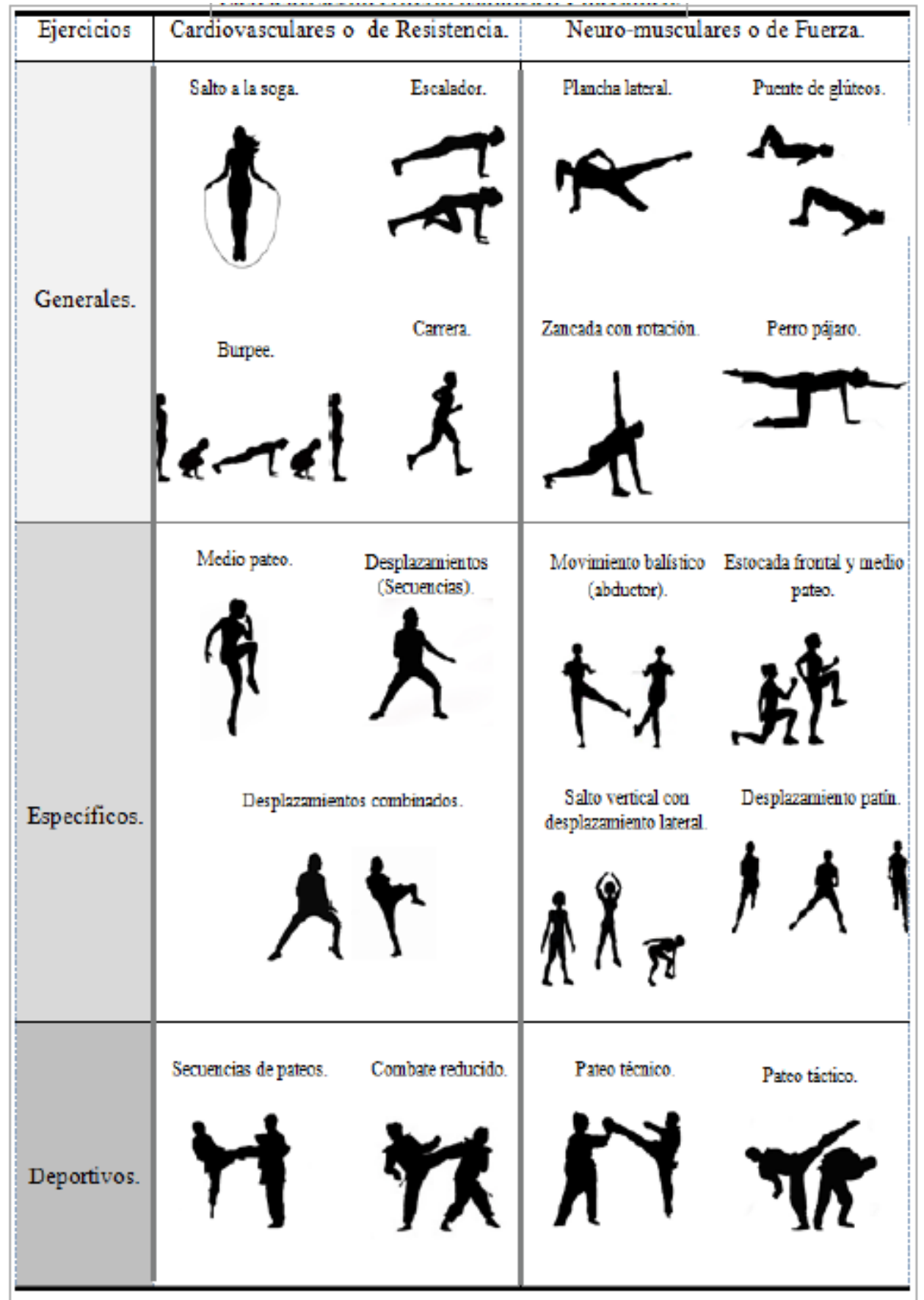


TABLA 2

\section{Breve descripción de los ejercicios}

\begin{tabular}{|c|c|}
\hline Nombre. & Descripción. \\
\hline Salto a la soga. & $\begin{array}{l}\text { Movimientos básicos de salto. Implementar diferentes variantes: } \\
\text { bipodales (alternado, pie juntos), unipodales, skipping, etc. }\end{array}$ \\
\hline Escalador. & $\begin{array}{l}\text { En posición decúbito ventral, brazos extendidos por debajo de } \\
\text { la línea de los hombros. En forma altemada (derecha / } \\
\text { izquierda) se realiza flexión de cadera y rodilla. }\end{array}$ \\
\hline Burpee. & $\begin{array}{c}\text { Desde posición de pie, se va a posición en cuclillas con las } \\
\text { manos sobre el suelo (1). Se extienden ambas piemas atrás y a } \\
\text { su vez se hace una flexión de codo. Se vuelve a la posición } \\
\text { número ly se realiza un salto vertical. }\end{array}$ \\
\hline Carrera. & $\begin{array}{l}\text { Se realizan traslaciones cortas utilizando la carrera como } \\
\text { motricidad general empleando el espacio del tatami. }\end{array}$ \\
\hline Plancha (lateral). & $\begin{array}{l}\text { En posición decúbito lateral, con el antebrazo en el suelo y el } \\
\text { codo debajo del hombro. El cuerpo elevado en línea recta desde } \\
\text { los tobillos, pasando por la cadera y hombros, hasta las orejas. }\end{array}$ \\
\hline Puente de glúteos. & $\begin{array}{l}\text { En posición decúbito dorsal, cadera y rodillas flexionadas. } \\
\text { Planta de los pies afirmados al suelo y separados el ancho de la } \\
\text { cadera. Elevar la cadera al mismo tiempo que se contraen los } \\
\text { glúteos. Tanto la cadera, los muslos y las rodillas deben estar } \\
\text { alineadas. Volver a la posición inicial. }\end{array}$ \\
\hline Zancada con rotación. & $\begin{array}{l}\text { Dar un paso adelante con la piema izquierda. La piema } \\
\text { contraria recta. Bajar el cuerpo hacia el suelo. Una vez en } \\
\text { posición de zancada, estirar el brazo derecho por encima de la } \\
\text { cabeza y hacia la izquierda. }\end{array}$ \\
\hline Perro pájaro. & $\begin{array}{l}\text { En posición de cuadrupedia. Manos debajo de la línea de los } \\
\text { hombros y rodillas debajo de la línea de la cadera. Extender } \\
\text { piema y brazo opuestos al mismo tiempo. Mantener } 3 \\
\text { segundos, regresar a la posición inicial e invertir. }\end{array}$ \\
\hline Medio pateo. & $\begin{array}{l}\text { De pie, con los pies separados un ancho de cadera. Flexionar la } \\
\text { cadera y la rodilla izquierda; al mismo tiempo flexionar codo } \\
\text { contrario en } 90^{\circ} \text {. Alterar de forma continua con la otra piema. }\end{array}$ \\
\hline $\begin{array}{l}\text { Desplazamientos } \\
\text { (secuencias) }\end{array}$ & $\begin{array}{l}\text { Incluir fintas o desplazamientos continuos, simples o } \\
\text { combinados, circulares o lineales, imaginando diferentes } \\
\text { acciones tácticas (ataque, contraataque, esquiva, afrontamiento) } \\
\text { sin efectuar pateos. }\end{array}$ \\
\hline $\begin{array}{l}\text { Desplazamientos } \\
\text { combinados. }\end{array}$ & $\begin{array}{c}\text { Ídem anterior, se incluyen medio pateo (explicado } \\
\text { anteriormente). }\end{array}$ \\
\hline $\begin{array}{l}\text { Movimiento balístico } \\
\text { (abductor) }\end{array}$ & Balancear la piema cerca del rango máximo de movimiento. \\
\hline $\begin{array}{l}\text { Estocada frontal y } \\
\text { medio pateo. }\end{array}$ & $\begin{array}{l}\text { En posición de pie y piemas ligeramente separadas. Adelantar } \\
\text { una piema. La otra flexiona la rodilla hasta quedar con la piema } \\
\text { paralela al suelo. Impulsarse con la piema de atrás realizando un } \\
\text { medio pateo. }\end{array}$ \\
\hline $\begin{array}{l}\text { Salto vertical con } \\
\text { desplazamiento lateral. }\end{array}$ & $\begin{array}{c}\text { De pie, con separación del ancho de cadera. Realizamos un } \\
\text { salto vertical. Una vez en contacto con el suelo desplazar hacia } \\
\text { un lateral. }\end{array}$ \\
\hline Desplazamiento patin. & $\begin{array}{l}\text { En posición de pie, se impulsa y estira de forma lateral la piema } \\
\text { izquierda cargando el peso del cuerpo. La piema derecha se } \\
\text { arrastra y realiza un medio pateo. Se repite la ejecución } \\
\text { invirtiendo el sentido. }\end{array}$ \\
\hline Secuencia de pateos. & $\begin{array}{l}\text { Un compañero sostiene un escudo o palmetas. Se realizan } \\
\text { diferentes secuencias de acciones tácticas (de 3-6 continuas). }\end{array}$ \\
\hline Combate reducido. & Se realizan combates de 30 segundos a 1 minuto. \\
\hline Pateo técnico. & $\begin{array}{c}\text { Se realizan distintas acciones técnicas de patada o puño (ap } \\
\text { chagui, furio chagui, yop chagui, etc.) }\end{array}$ \\
\hline Pateo táctico. & $\begin{array}{l}\text { Se realiza aplicaciones inteligentes de las acciones técnicas con } \\
\text { el compañero, escudo, palmeta u otros elementos. }\end{array}$ \\
\hline
\end{tabular}




\section{CONSIDERACIONES FINALES Y APLICACIONES PRÁCTICAS.}

El APD es un componente esencial para el rendimiento y, como tal, debe ser tenido en cuenta tanto para el entrenamiento como la competencia. Son numerosos los estudios que analizaron los efectos de rutinas de ejercicios de estiramiento muscular, dentro del APD, sobre el rendimiento. Otros estudios, se ocuparon de examinar la importancia de la duración, intensidad y combinación de diferentes técnicas, para optimizar el desempeño en entrenamiento y competencia. También, las investigaciones propusieron modelos de APD para diferentes disciplinas. Sin embargo, este es el primer trabajo académico que propone, desde el análisis de los ejercicios y con criterios científicos, un APD específico para el Taekwondo Olímpico.

El APD es una actividad fundamental para el rendimiento en el Taekwondo Olímpico, contribuyendo a configurar y potenciar la carga de entrenamiento y/o competición.

Metodológicamente, el acondicionamiento previo deportivo se desarrolla en la parte inicial de la sesión de entrenamiento y/o de competición. Se estructura en tres fases bien definidas que incluyen ejercicios tanto cardiovasculares como neuromusculares, que, a su vez, son generales, específicos y deportivos, según su correspondencia mecánica con las acciones del Taekwondo Olímpico.

También, es importante destacar el uso de la progresión, en dificultad e intensidad, de los ejercicios dentro del APD, y la duración total de la actividad. Finalmente, se deben realizar más estudios e investigaciones para contribuir al desarrollo de estas primeras proposiciones metodológicas. Y, lejos de generalizarlas, profundizarlas y enriquecerlas para una mejor práctica de este deporte.

\section{Bibliografía.}

American College Sports Medicine, ACSM (2012). Foundations of strength training and conditioning. United States. (Primera Edición) Lippincott, Williams \& Wilkins.

Berger, J. \& Hauptman, M. (1996). La clasificación de los ejercicios físicos. RED, IX, (3): 31-37. En: González Ravé, J. M., Navarro Valdivieso F., Delgado Fernández, M, García García, J. M. (2010). Fundamentos del entrenamiento deportivo. Sevilla, España. Wanceulen.

Bridge, C. A., Ferreira da Silva Santos, J., Chaabéne, H., Pieter, W. \& Franchini, E. (2014). Physical and physiological profiles of Taekwondo athletes. Sports Medicine, 44: 713-733.

Bompa, T. O. (1993). Periodization of strength training: the new wave in strength training. Toronto, Canadá. Veritas Publishing.

Casas, A. (Mayo, 2008) Acondicionamiento previo deportivo y prevención de lesiones. Cuadernillo de Master en Ejercicio Preventivo y Terapéutico de Universidad Europea de Madrid (pp.14-44). Villaviciosa de Odón, Madrid, España.

Coffey, V. G. \& Hawley, J. A. (2007). The molecular bases of training adaptation. Sports Medicine 37, 737-763.

Derenne, C., Ho, K. W. \& Murphy, J. C. (2001). Effects of general, special and specific resistance training on throwing velocity in baseball: a brief review. Journal of Strength and Conditioning Research 15 (1):148-156.

González Ravé, J. M., Navarro Valdivieso F., Delgado Fernández, M \& García García, J. M. (2010). Fundamentos del entrenamiento deportivo. Sevilla, España. Wanceulen.

Harre, D. (1987). Teoría del entrenamiento deportivo. Ed. Stadium. Buenos Aires. En: González Ravé, J. M., Navarro Valdivieso F., Delgado Fernández, M, García García, J. M. (2010). Fundamentos del entrenamiento deportivo. Sevilla, España. Wanceulen.

Kuznetsov, V. (1975). Metodología del entrenamiento de la fuerza para deportistas de alto nivel. Buenos Aires, Argentina. Stadium.

Manso, J. M. G., Valdivielso, M. N., \& Caballero, J. A. R. (1996). Planificación del entrenamiento deportivo. Madrid. España. Gymnos. 
Matveiev, L. P. (1985). Fundamentos del entrenamiento deportivo. Moscú, Rusia. Eduga. En: Manso, J. M. G., Valdivielso, M. N., \& Caballero, J. A. R. (1996). Planificación del entrenamiento deportivo. Gymnos.

Siff, M. C. \& Verhoshansky, Y. (2004). Superentrenamiento. España. Paidotribo. 